\title{
Editorial
}

\section{Radiotracer Techniques: A Unique Tool in Marine Ecotoxicological Studies}

This special issue of Environmental Bioindicators contains a selection of papers presented during a session co-chaired by the authors at the Fifteenth ISEBI International Conference on Environmental Bioindicators, which took place in the City University of Hong-Kong (Kowloon, Hong-Kong) on 7-9 June 2007.

The theme of this special issue focuses on the use of radiotracer techniques to investigate the behaviour and fate of contaminants, including radionuclides, metals, organic pollutants and biotoxins in marine coastal environments, and the link between their environmental chemistry and organism physiology in both laboratory and field studies.

In comparison to classical approaches, highly sensitive nuclear techniques offer several unique advantages, such as cost effectiveness and elevated throughput of samples. Gammaemitting radiotracers also allow radioanalysis of live organisms over relatively long observation periods. This characteristic allows data to be generated with reduced biological variability and substantially decreases the number of experimental organisms that need to be sacrificed. Because of their extremely high sensitivity, nuclear applications also enable bioaccumulation experiments to be conducted under realistic exposure conditions, i.e. using low, naturally-occurring concentrations of radiolabelled pollutants. In particular, radiotracer techniques provide unique and powerful tools to study pollutant bioaccumulation kinetics and to determine key parameters relating to the target organisms, such as pollutant uptake and depuration rates, assimilation efficiencies and biological half-lives. Radiotracer techniques also aid in the formalisation of some key mechanisms driving bioaccumulation and the relative contribution of different contamination pathways (e.g., water, food, sediment) to the 
global bioaccumulation process of pollutants. Owing to these unique advantages, radiotracer techniques are widely recognised as the cutting edge of experimental marine ecotoxicology.

These few characteristics outlined above, which by no means paint a comprehensive picture of all the advantages of radiotracer techniques, already demonstrate that nuclear applications are invaluable from both the sensitivity and the cost-effective viewpoints. They can provide reliable information over much shorter periods than is possible using conventional stable isotope techniques. Radiotracer techniques are particularly useful when rapid results are required concerning environmental issues, guidelines and surveys associated with nuclear technologies such as nuclear power plants, or with regard to conventional pollutants such as metals and organics in seafood or the characterization and validation of bioindicator species.

The International Atomic Energy Agency (IAEA) promotes the sharing of knowledge of peaceful nuclear applications in environmental studies across the world. To this end, the IAEA supports technical cooperation (TC) projects and coordinated research projects (CRP) to enhance knowledge and expertise in less developed Member States, through capacity building and well organised mechanisms of knowledge transfer. Using national, regional and inter-regional projects, including technical training, training courses, capacity building and expert missions, the IAEA Technical Cooperation Programme offers countries important scientific assistance to enhance environmental and bioindicator studies using radio-isotopic techniques. By coupling the technical and management expertise of its five Departments, the IAEA has succeeded in generating a powerful and highly efficient way to support its International Cooperation Programme for over five decades (http://www.iaea.org/OurWork/index.html).

The papers presented in this special issue of Environmental Bioindicators are good examples of some of the recent achievements of the IAEA International Cooperation 
Programme. They result from collaboration between the Department of Nuclear Sciences and Applications, the Department of Technical Cooperation and IAEA Member States. Papers were invited from teams supported by CRP or TC projects - teams that only recently became involved in ecotoxicological radiotracer techniques, but that have already reached proficiency. For instance, the CEAC (Centro de Estudios Ambientales di Cienfuegos, Cuba) is now a recognised Resource Centre for the whole Caribbean region.

Among the papers presented here, two focus on the transfer of a highly toxic organic pollutant (hexachlorobenzene, HCB) from sediment to edible bivalves from the Brazilian coasts, addressing uptake kinetics and retention of HCB within these molluscs (both papers by de Andréa and co-authors). One paper (Gómez-Batista and co-authors) is dedicated to the selection and validation of reliable sentinel species for the monitoring of arsenic contamination levels in Cienfuegos Bay (Cuba), where a major arsenic spill occurred in late 2001. This spill prevented consumption of any local seafood and fish by the local population of 150,000 inhabitants for several months. The paper by Qureshi and co-authors reports on the influence of environmental parameters on the bioaccumulation of radionuclides and metals in mussels from the coast of Pakistan. Important nuclear facilities are located along this coast, stressing the need to develop and implement a local biomonitoring program. The Srisuksawad and Prasertchiewchan paper investigates the bioaccumulation of toxic metals in bivalves in order to contribute to strengthening the monitoring program of Thailand, where many fisheryrelated activities are carried out. Finally, the paper by Sombrito and co-authors reports on a state-of-the art nuclear detection technique to assess levels of algal biotoxins in edible seafood exposed to harmful algal blooms (HABs), which are recognised among today's major seafood safety and socio-economic global issues.

The guest editor (MW) is most grateful to all the anonymous reviewers who took part in the revision process of this special issue for their invaluable contribution. He also would like 
to thank the Editors-in-Chief of Environmental Bioindicators, Ed Zillioux and Jim Newman, for their confidence and support throughout the editing process.

Michel WARNAU ${ }^{1}$ and Paco BUSTAMANTE ${ }^{2}$

${ }^{1}$ IAEA - Marine Environment Laboratories, Principality of Monaco

${ }^{2}$ University of La Rochelle - CRELA, France 